\title{
Sensitive methods for the detection of an insertion in exon 20 of the HER2 gene in the metastasis of non-small cell lung cancer to the central nervous system
}

\author{
PAWEŁ KRAWCZYK ${ }^{1}$, MARCIN NICOŚ ${ }^{1,2}$, TOMASZ POWRÓZEK ${ }^{1}$, RADOSŁAW MLAK ${ }^{1}$, \\ MAREK SAWICKI ${ }^{3}$, BOŻENA JAROSZ ${ }^{4}$, BEATA PAJĄK ${ }^{5,6}$, KRZYSZTOF KUCHARCZYK ${ }^{5}$, \\ DARIUSZ STENCEL $^{7}$, TOMASZ TROJANOWSKI ${ }^{4}$ and JANUSZ MILANOWSKI ${ }^{1,8}$
}

${ }^{1}$ Department of Pneumonology, Oncology and Allergology, Medical University of Lublin, Lubin 20-954;

${ }^{2}$ Postgraduate School of Molecular Medicine, Warsaw 02-091;

Departments of ${ }^{3}$ Thoracic Surgery and ${ }^{4}$ Neurosurgery and Pediatric Neurosurgery, Medical University of Lublin, Lubin 20-954; ${ }^{5}$ BioVectis Ltd., Kucharczyk TE, Warsaw 02-106; ${ }^{6}$ Electron Microscopy Platform,

Mossakowski Medical Research Centre, Polish Academy of Sciences, Warsaw 02-106;

${ }^{7}$ Boehringer Ingelheim Poland, Warsaw 02-675; ${ }^{8}$ Institute of Agricultural Medicine, Lublin 20-090, Poland

Received January 14, 2013; Accepted June 27, 2013

DOI: $10.3892 / 01.2013 .1495$

\begin{abstract}
The HER2 (ErbB2/neu) protein is a member of the HER (ErbB) receptor family (EGFR, HER2, HER3 and HER4) that expresses tyrosine kinase activity in the intracellular domain. EGFR and HER2 overexpression is observed in numerous types of cancer, nevertheless, the susceptibility of patients with non-small cell lung cancer (NSCLC) to therapy with EGFR and HER2 tyrosine kinase inhibitors (TKIs) depends on mutations present in the respective coding genes (driver mutations). In the present study, PCR and amplified DNA fragment length analysis (FLA) were used along with the multi-temperature single-strand conformation polymorphism (MSSCP) technique in order to identify the 12 base pair insertion in exon 20 of the HER2 gene in 143 patients with NSCLC metastasis to the central nervous system. The prevalence of the HER 2 gene mutation was correlated with mutations in the EGFR and BRAF genes. The insertion in exon 20 of the HER2 gene was observed in a single 77-year-old, non-smoking male, with poorly-differentiated adenocarcinoma of the lung (1.5\% of adenocarcinoma patients). No other genetic abnormalities were identified in this patient. In the therapy of NSCLC patients with HER2 gene mutations, drugs that inhibit the EGFR and HER2 receptors, for example
\end{abstract}

Correspondence to: Professor Paweł Krawczyk, Immunology and Genetics Laboratory, Department of Pneumonology, Oncology and Allergology, Medical University of Lublin, 8 Jaczewskiego, Lublin 20-954, Poland

E-mail: krapa@poczta.onet.pl

Key words: non-small cell lung cancer, metastasis to the central nervous system, HER2 mutation afatinib, may be effective. The identification of other driving mutations in NSCLC cells appears to be key to the appropriate qualification of molecular targeted therapies.

\section{Introduction}

The HER (ErbB) receptor family consists of four receptors: HER1 (EGFR, ErbB1), HER2 (Neu, ErbB2), HER3 (ErbB3) and HER4 (ErbB4) that express tyrosine kinase activity in the intracellular domain. The ErbB abbreviation derives from the erythroblastic leukemia oncogenic virus (erythroblastic leukemia viral oncogene), which is structurally homologous to human HER receptors (1). The signal for epithelial cell proliferation is transduced to the cell nucleus as a result of the homo- and heterodimerization of HER receptors and activation of the complex by corresponding ligand binding. The preferred partner for EGFR heterodimerization is the HER2 receptor, which is also known to be overexpressed in breast cancer (2). Mutations within the tyrosine kinase domain of the EGFR gene, mainly deletions in exon 19 or substitution L858R in exon 21, are detected in $~ 10 \%$ of Caucasian patients with non-small cell lung cancer (NSCLC) (3). Additionally, the $E G F R$ gene mutation affects the efficacy of $E G F R$ tyrosine kinase inhibitors (TKIs). There is certain evidence that HER2 gene mutations, and perhaps the high expression of the HER2 receptor, may be involved in the etiology of certain NSCLC cases (4).

Mutations in the HER2 gene tyrosine kinase domain are extremely rare in NSCLC patients (5). Preliminary data show that the prevalence is not higher than $2 \%$ in the general patient population (6). The mutations are most often indicated in non-smoking females with adenocarcinoma of the lung (7). The most significant mutations are two different insertions of 12 base pairs, which impair the reading frame in exon 20 of the HER2 gene: A775YVMA (66\% of all detected mutations 
in the HER2 gene) or M774AYVM. These mutations are identical to the insertion of nine base pairs in exon 20 of the EGFR gene, which makes the structure of the tyrosine kinase domain of the HER2 protein similar to the structure of the tyrosine kinase domain of the EGFR gene, modified by the mentioned mutations (5). Based on this, it is assumed that A775YVMA or M774AYVM mutations of the HER2 gene cause similar consequences to the mutations in exon 20 of the EGFR gene (8). Taking into account that the HER2 receptor predominantly undergoes heterodimerization with EGFR, the narrowing of the binding pocket for ATP, resulting from a mutation in exon 20 of the EGFR or HER2 genes in heterodimer EGFR/ HER2, leads to the increased activity of the tyrosine kinases of those receptors (9). This results in an increase of the phosphorylation of further signal proteins, cancer cell proliferation and resistance (or declined susceptibility) to reversible EGFR TKIs (10).

In the future, the presence of the A775YVMA or M774AYVM mutations in the HER2 gene may be a potential predictive marker of effectiveness of HER family TKIs and be a target for new molecular targeted therapies. The detection of an insertion in exon 20 of the HER 2 gene may play a role in therapy design, and be as important as the current assessment for T790M mutations in the EGFR gene, which appears to be a main cause of resistance for reversible EGFR TKIs (50\% of all acquired resistance). It should be noted that the brain is the most frequent location for metastases of lung adenocarcinoma. However, there is limited evidence on the prevalence of HER2 gene mutations in metastatic NSCLC and in patients with histologies other than adenocarcinoma.

\section{Materials and methods}

Patients. The present study retrospectively analyzed 143 patients (99 male and 44 female) ranging in age between 38 and 81 years (59.8 \pm 8.8 years), for whom paraffin-embedded cancer tissue from NSCLC metastatic lesions in the brain was available. The patients underwent routine neurosurgical procedures with a palliative aim. In 32 patients, material from the primary tumor, obtained during thoracoscopy, intrabronchial, transbronchial or transthoracic biopsy, was available. Written informed consent was obtained from all patients. This study was approved by the Ethics Committee of the Medical University of Lublin, Poland (No. KE-0254/131/2011).

Lung adenocarcinoma was diagnosed in 61 patients $(42.6 \%)$. Squamous and large cell carcinomas were confirmed in $23(16.1 \%)$ and $21(14.7 \%)$ cases, respectively. In 38 patients (26.6\%) the NSCLC subtype was impossible to assess and they were diagnosed as not otherwise specified (NOS). The median survival time from lung cancer diagnosis to death was 9.2 months. None of the patients were treateadted with EGFR TKI (EGFR TKI naïve).

Identification of mutations. DNA was isolated from paraffin-embedded material containing metastatic lesions and from primary tumor tissues using a QIAamp DNA FFPE tissue kit (Qiagen, Valencia, CA, USA). Estimation of the insertion in exon 20 of the HER 2 gene was conducted using a PCR reaction with primers flanking the mutated region of the HER2 gene. Primers were fluorescently labeled (Cy5). Amplified
DNA fragment length analysis (DNA-FLA) was applied using an ALF Express II sequencer (Amersham Pharmacia Biotech AB, Uppsala, Sweden) in polyacrylamide gel. The HER2 gene mutation was also confirmed by the native electrophoretic separation multi-temperature single-strand conformation polymorphism (MSSCP) technique (11), which allows separation of the different conformers of single-stranded (ss)DNA fragments, in order to differentiate wild-type HER2 from mutated-type HER2. In the MSSCP-based minor variant enrichment procedure, the PCR products were analyzed at strictly controlled temperatures $\left( \pm 0.2^{\circ} \mathrm{C}\right)$ using dedicated equipment, the DNAPointer ${ }^{\circledR}$ System (BioVectis, Warsaw, Poland), as described by Kaczanowski et al (11). In general, the PCR products were heat denatured and ssDNA conformers were resolved on $9 \%$ polyacrylamide gel with $5 \%$ glycerol in native conditions (TBE buffer) at three different temperatures $\left(35,25\right.$ and $\left.15^{\circ} \mathrm{C}\right)$ during one run. Subsequently, the DNA bands were visualized by silver nitrate staining (SilverStain DNA kit; BioVectis). Fragments of the MSSCP gel containing the bands of interest were cut out and the SsDNA was eluted and re-amplified using primers and PCR conditions as described previously. For subsequent DNA Sanger sequencing (12) a 1/10 volume of obtained PCR products was used (3730xl DNA Analyzer, Applied Biosystems, Carlsbad, CA, USA).

Additionally, the deletion in exon 19, substitution L858R in the EGFR gene and mutation V600E in the BRAF gene, were assessed in the analyzed material.

\section{Results}

The insertion in exon 20 of the HER2 gene (insertion version A775YVMA or M774AYVM) was indicated in a single patient, who only had material from the NSCLC metastatic lesion available $(0.67 \%$ of all analyzed patients and $1.5 \%$ of adenocarcinoma patients; Fig. 1). The mutation was confirmed by the MSSCP technique (Fig. 2). The patient was a 77-year-old non-smoking male, with advanced poorly-differentiated lung adenocarcinoma with metastases in the cerebellum. Due to the low performance status following neurosurgery, and taking into account the advanced stage of the disease, the patient was not treated with chemotherapy, radiotherapy or molecular targeted therapy. Additionally, molecular assessments led to exclusion of other activating mutations in exons 19, 20 (T790M) and 21 (L858R) of the EGFR gene, as well as V600E substitution in the BRAF gene in the patient with the HER2 mutation.

In total, 9 activating mutations $(6.3 \%)$ of the EGFR gene were detected in brain samples, these consisted of three delE746-A750 deletions of 15 base pairs in exon $19(2.1 \%)$ and six with L858R substitutions in exon 21 (4.2\%). Two deletions in exon 19 were detected in giant cell carcinoma patients and one in an adenocarcinoma patient, while all L858R substitutions were diagnosed in adenocarcinoma patients. Evaluation of the primary tumors revealed EGFR mutations similar to those in corresponding metastases: One delE746-A750 deletion in exon 19 and one L858R substitution in exon 21. Additionally, V600E substitutions in the $B R A F$ gene were not detected in the brain metastases of the NSCLC patients.

Additionally, the results obtained using PCR analysis were verified with the use of the MSSCP method. As shown 


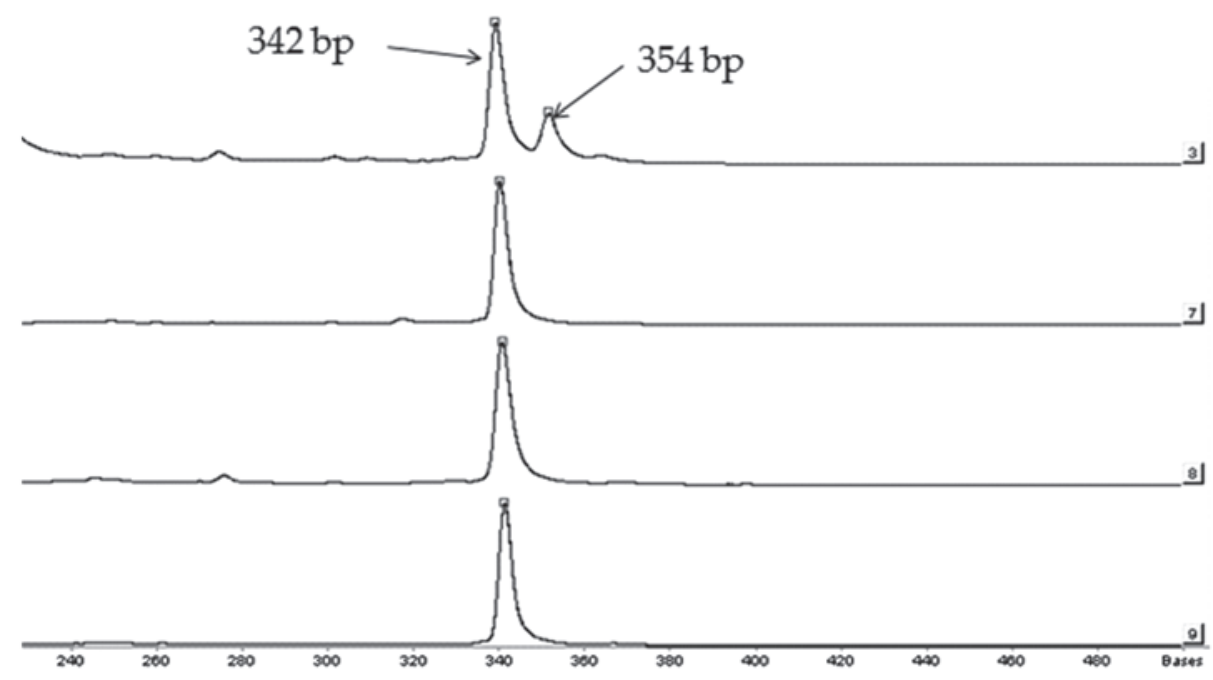

Figure 1. Example of HER2 gene mutation analysis. The double peaks on path 3 refer to two PCR products, differing in base pair number: The shorter, consisting of 342 base pairs, is typical for the HER2 wild-type (WT) gene and the longer (insertion of 12 base pairs), is characteristic for mutated (MT) HER2. The single peak, containing 342 base pairs on paths 7-9 indicates the presence of only WT HER 2 genes.

A

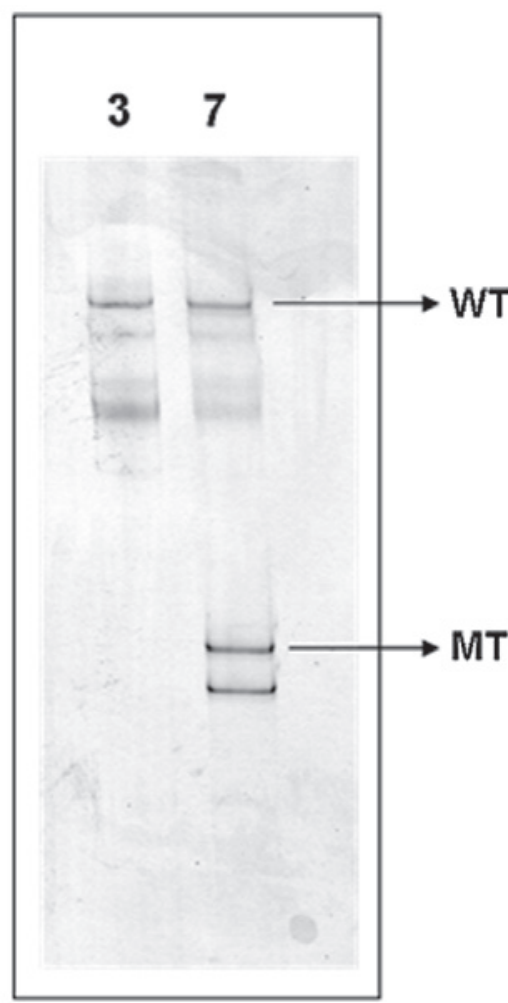

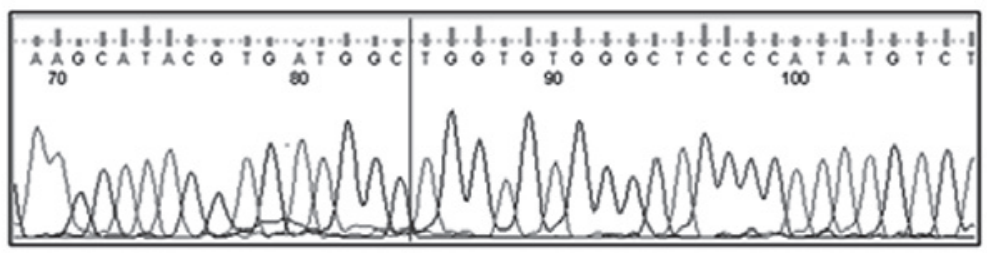

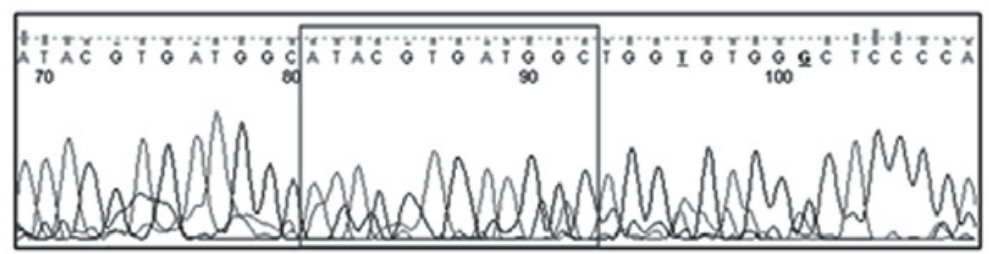

C

\begin{tabular}{|c|c|c|c|c|c|c|c|c|}
\hline & 1 & 11 & 21 & 31 & 41 & 51 & 61 & 71 \\
\hline NER2UT & \multirow{2}{*}{\multicolumn{8}{|c|}{ 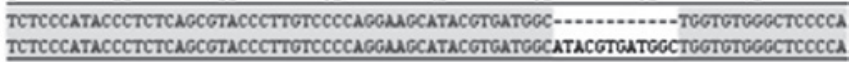 }} \\
\hline VERENT & & & & & & & & \\
\hline \multirow[t]{2}{*}{ Consensus } & \multicolumn{6}{|c|}{ teteccataceeteteagegtaceetrgtececaggasgeatacgtgatgge } & \multicolumn{2}{|c|}{ tgrtgtgggetececa } \\
\hline & 81 & 91 & 101 & 111 & 121 & 131 & 141 & 151 \\
\hline MER2WT & TATE & $\mathrm{cacct}$ & GCAT & $\operatorname{GACA}$ & $\operatorname{GrecA}$ & Taxa: & TATEC & Gөстесететт \\
\hline NERREIT & TATE & $\operatorname{coccl}$ & CCAT & $\operatorname{GACA}$ & $\operatorname{GrecA}$ & $\mathrm{Tanc}$ & TATEC & QCCTRCCTCTIT \\
\hline \multirow[t]{2}{*}{ Consensus } & \multicolumn{8}{|c|}{ 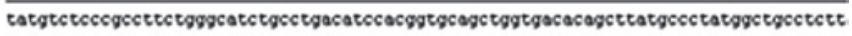 } \\
\hline & 161 & 171 & 181 & 191 & 201 & 211 & 221 & 231 \\
\hline NERZUT & \multirow{2}{*}{\multicolumn{8}{|c|}{ 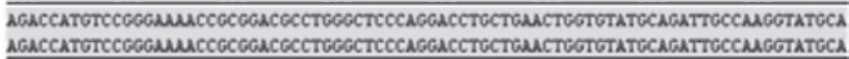 }} \\
\hline VEREIT & & & & & & & & \\
\hline \multirow[t]{2}{*}{ Consensus } & \multicolumn{8}{|c|}{ agaceargrecgggasacegeggacgectgggeteceaggacetgergaserggrgratgeagactgecanggratgea } \\
\hline & 241 & 251 & 261 & 271 & 281 & 291 & 301 & 311 \\
\hline $\begin{array}{l}\text { MERTWT } \\
\text { MER2IIT }\end{array}$ & $\begin{array}{l}\text { CCTE } \\
\text { CCTE }\end{array}$ & $\begin{array}{l}\text { TrToC } \\
\text { TrToC }\end{array}$ & $\begin{array}{l}\text { тете } \\
\text { тете }\end{array}$ & $\begin{array}{l}\mathrm{wucc} \\
\mathrm{wec}\end{array}$ & $\begin{array}{l}\mathrm{GTCCA} \\
\mathrm{GTCCA}\end{array}$ & $\begin{array}{l}\text { COCCT: } \\
\text { COCCT }\end{array}$ & & \\
\hline
\end{tabular}

Figure 2. MSSCP electrophoresis easily differentiates between two genetic variants of HER2. (A) MSSCP electrophoretic profiles of samples 72 (line 3) and 7 (line 7). PCR products of exon 20 HER2 gene amplification were heat denatured and ssDNA was separated on $9 \%$ polyacrylamide gel using the MSSCP method under optimal electrophoretic conditions. DNA bands were visualized with silver stain. ssDNA bands corresponding to wild-type (WT) and mutated (MT) sequences are indicated by arrows. (B) Partial DNA chromatograms showing the presence of 12 nucleotide insertions in the DNA sequence obtained from the ssDNA band representing mutated HER2 amplicon (frame). The line in the WT partial DNA chromatogram points to the lack of sequence characteristics for the mutated genetic variant. (C) Sequence alignment of WT and MT HER2 amplicons detected in sample 7. The 12 nucleotide insertion is visible. MSSCP, multi-temperature single-strand conformation polymorphism; ssDNA, single-stranded DNA. 
in Fig. 2, MSSCP separation of exon 20 HER2 sample numbers 72 (line 3) and 7 (line 7), recognized previously as the wild-type (WT) and mutated variant (MT), respectively, revealed their distinct electrophoretic profiles. Sample 7 contained additional ssDNA bands, which were not observed in sample 72 (Fig. 2A). It was assumed that similar ssDNA bands detected in each of the two samples indicated the WT HER2 genetic variant. Considering that the MSSCP method is based on non-denaturing polyacrylamide electrophoresis, additional ssDNA bands suggested the presence of DNA conformers, representing additional amplicon sequences. To verify this hypothesis, indicated ssDNA bands were cut out from the gel and the DNA was recovered, re-amplified and Sanger sequenced. Further analysis of the obtained DNA sequences confirmed our suppositions (Fig. 2B). According to the BLAST database, the ssDNA bands observed in samples 72 and 7, corresponded to the WT HER2 sequence. Additional ssDNA bands in sample 7 contained the mutated HER 2 sequence with a 12-nucleotide insertion. The described results fully confirmed that sample number 7 was a mixture of two genetic variants of the HER2 amplicon. The comparison of the WT and the MT DNA sequence detected in sample 7 is illustrated in Fig. 2C.

\section{Discussion}

The present study has shown that primary HER2 gene mutations are detectable in Caucasian patients with NSCLC. Furthermore, HER2 gene mutations were indicated in metastatic lesions of lung cancer in the cerebellum, which, to the best of our knowledge is the first report of this worldwide. HER2 gene mutations are believed to be responsible for the development of these lung cancer types, which occur independent of smoking and are mainly adenocarcinomas. However, the prevalence of HER 2 gene mutations in Caucasian patients is extremely low; in the present study, it has been recorded as occurring in $<1 \%$ of patients with NSCLC.

Shigematsu et al have searched for HER2 gene mutations in 671 primary NSCLC tumors and 80 NSCLC cell lines, as well as in other types of cancer (14). The authors identified different insertions in exon 20 of the HER 2 gene in 11 patients with NSCLC (1.6\%) and in one lung adenocarcinoma cell line (NCI-H1781). This mutation was not observed in other types of cancer, including 55 SCLC tumors. HER2 gene mutations were more frequent in non-smokers $(3.2 \% ; 8 / 248)$ and were present solely in patients with adenocarcinomas $(2.8 \% ; 11 / 394)$. Additionally they were more frequent in female $(2.7 \%$; 7/258) compared with male $(1 \% ; 4 / 413)$ patients. Notably, only one mutation was diagnosed in Caucasian patients $(0.7 \%$; 1/137) (5). In another study of 95 patients with NSCLC, Sasaki et al described only one non-smoking female patient with lung adenocarcinoma harboring an insertion of 12 nucleotides in exon 20 of the HER2 gene (6). Buttitta et al diagnosed HER2 gene mutations in 9 out of 403 Caucasian patients with lung adenocarcinoma (2.2\%), but only 7 mutations were determined to be an insertion of 12 nucleotide pairs in exon 20. Mutations were more frequent in female (4.1\%) and non-smoking $(3.1 \%)$ patients with bronchioalveolar cancer $(6.2 \%)$, however, they were also indicated in male patients $(1.8 \%)$ and smokers (1.9\%) (7).
The data from the aforementioned studies, as well as that from the present study, are contrary to the data of Stephen et al, which showed that mutations in the tyrosine kinase domain of the HER2 gene (different HER2 mutations and abnormalities) are observed in up to $4 \%$ of patients with NSCLC (120 primary NSCLC tumors tested), of which, $10 \%$ of patients had adenocarcinomas (8). Moreover, Li et al described as many as 12 HER2 gene mutation carriers in a group of 202 (6\%) non-smoking Asian patients who underwent surgery for treatment of lung adenocarcinomas (10).

Assuming that HER2 gene mutations are so-called driver mutations, which drive the epithelial cells into carcinogenesis, and that they do not coexist with other driver mutations (in the present article the coincidence of insertions in exon 20 of the HER2 gene with EGFR and BRAF gene mutations was not confirmed), patients with these mutations may require specifically targeted therapy against the tyrosine kinase of HER2. However, TKIs that inhibit only EGFR tyrosine kinase activity (gefitinib and erlotinib) in cases of only HER2 gene mutation are ineffective. The reversible, dual EGFR and HER2 tyrosine kinase inhibitor, lapatinib, has showed certain activity in cell lines, but this is not clinically relevant in patients with NSCLC $(13,14)$. The irreversible ErbB family receptor blocker, afatinib (BIBW 2992), which inhibits EGFR, HER2 and HER4 tyrosine kinases, has been shown to be effective in the elimination of cancer cells with HER2 gene mutations in cell lines and animal models (15-19). Moreover, the first case reports of afatinib effectiveness in female patients with lung adenocarcinoma who are carriers of HER 2 gene mutations have been presented (20). There are ongoing in vitro experiments evaluating the feasibility of the use of combined therapy with afatinib and sirolimus (an mTOR inhibitor) in patients with HER2 gene mutations (4). Moreover, trastuzumab, a monoclonal antibody directed against the extracellular HER2 domain, which has effectively been used in breast cancer patients with HER2 overexpression, may be effective in patients with NSCLC that harbor HER2 gene mutations (21).

Based on the overall data we may conclude that gene profile analysis in cancer patients may extend the scope of molecular therapies used in patients with NSCLC. Moreover, in the near future, the personalized therapy of NSCLC based on the assessment of numerous different gene mutations in cancer cells may become a reality.

\section{Acknowledgements}

This study was partially funded by the European Community's Seventh Framework Programme (FP7/2007-2013) under the grant agreement in HEALTH-F2-2010-258677.

\section{References}

1. Hirsch FR, Varella-Garcia M and Cappuzzo F: Predictive value of EGFR and HER 2 overexpression in advanced non-small lung cancer. Oncogene 28: 32-37, 2009.

2. Sun M, Behrens C, Feng L, et al: HER family receptor abnormalities in lung cancer brain metastases and corresponding primary tumors. Clin Cancer Res 15: 4829-4837, 2009.

3. Mounawar M, Mukeria A, Le Calvez F, et al: Patters of EGFR, HER2, TP53, and KRAS mutation of p14arf expression in non-small lung cancer in relation to smoking history. Cancer Res 67: 5667-5672, 2007. 
4. Pao W and Girard N: New driver mutations in non-small-cell lung cancer. Lancet Oncol 12: 175-180, 2011.

5. Shigematsu H, Takahashi T, Nomura M, et al: Somatic mutations of the HER2 kinase domain in lung adenocarcinomas. Cancer Res 65: 1642-1646, 2005.

6. Sasaki H, Shimizu S, Endo K, et al: EGFR and erbB2 mutation status in Japanese lung cancer patients. Int J Cancer 118: 180-184, 2006.

7. Buttitta F, Barassi F, Fresu G, et al: Mutational analysis of the HER2 gene in lung tumors from Caucasian patients: mutations are mainly present in adenocarcinomas with bronchioloalveolar features. Int J Cancer 119: 2586-2591, 2006.

8. Stephen P, Hunter C, Bignell G, et al: Lung cancer: intragenic ERBB2 kinase mutations in tumours. Nature 431: 525-526, 2004

9. Wang SE, Narasanna A, Perez-Torres M, et al: HER2 kinase domain mutation results in constitutive phosphorylation and activation of HER 2 and EGFR and resistance to EGFR tyrosine kinase inhibitors. Cancer Cell 10: 25-38, 2006

10. Li C, Fang R, Sun Y, et al: Spectrum of oncogenic driver mutations in lung adenocarcinomas from east asian never smokers. PLoS One 6: e28204, 2011.

11. Kaczanowski R, Trzeciak L and Kucharczyk K: Multitemperature single-strand conformation polymorphism. Electrophoresis 22 $3539-3545,2001$

12. Sanger F, Nicklen S and Coulson AR: DNA sequencing with chain terminating inhibitors. Proc Natl Acad Sci 74: 5463-5467, 1977.

13. Diaz R, Nguewa PA, Parrondo R, et al: Antitumor and angiogenic effect of the dual EGFR and HER-2 tyrosine kinase inhibitor lapatinib in a lung cancer model. BMC Cancer 10: 188-197, 2010
14. Shimamura T, Ji H, Minami Y, et al: Non-small-cell lung cancer and $\mathrm{Ba} / \mathrm{F} 3$ transformed cells harboring the ERBB2 G776insV G/C mutation are sensitive to the dual-specific epidermal growth factor receptor and ERBB2 inhibitor HKI-272. Cancer Res 66: 6487-6491, 2006.

15. Reid A, Vidal L, Shaw $\mathrm{H}$ and de Bono J: Dual inhibition of ErbB1 (EGFR/HER1) and ErbB2 (HER2/neu). Eur J Cancer 43: 481-489, 2007.

16. Li D, Ambrogio L, Shimamura T, et al: BIBW2992, an irreversible EGFR/HER2 inhibitor highly effective in preclinical lung cancer models. Oncogene 27: 4702-4711, 2008.

17. Perera SA, Li D, Shimamura T, et al: HER2YVMA drives rapid development of adenosquamous lung tumors in mice that are sensitive to BIBW2992 and rapamycin combination therapy. Proc Natl Acad Sci USA 106: 474-479, 2009.

18. Shimamura T, Greulich H, Solca FF and Wong KK: Efficacy of BIBW-2992, potent irreversible inhibitor of EGFR and HER2 in human NSCLC xenografts in a transgenic mouse lung-cancer model: C7-04. J Thorac Oncol 2 (suppl): S380, 2007.

19. Hirsh V: Afatinib (BIBW 2992) development in non-small-cell lung cancer. Future Oncol 7: 817-825, 2011.

20. De Greve J, Teugels E, De Mey J, et al: Clinical activity of BIBW2992, an irreversible inhibitor of EGFR and HER2 in adenocarcinoma of the lung with mutations in the kinase domain of HER2neu. J Thorac Oncol 4: S307 (abstr), 2009.

21. Heinmöller P, Gross C, Beyser K, et al: HER2 status in non-small cell lung cancer: results from patient screening for enrollment to a phase II study of herceptin. Clin Cancer Res 9: 5238-5243, 2003. 